\title{
ECHELLE SPECTROSCOPY WITH A CCD AT LOW SIGNAL-TO-NOISE RATIO
}

\author{
Didier Queloz
}

Observatoire de Genève

\begin{abstract}
The measurement of some physical parameters of astronomical objects can only be carried out with high resolution spectra. Unfortunately the high dispersion of the light on the detector restricts such observations to relatively bright sources. However, some spectral information can be concentrated into a single spectral "line" by a cross-correlation algorithm, allowing the observation of fainter objects. Such a technique, taken from the CORAVEL optical correlation, is presented. A complete description of the errors of the correlation function parameters is given and the minimum signal-to-noise ratio is also discussed. Finally, a short investigation of the best resolution needed to observe efficiently radial velocities and velocity broadenings is made.
\end{abstract}

\section{INTRODUCTION}

The measurement of some physical parameters from stellar spectra, such as accurate radial velocities, broadenings of slow rotators or intrinsic velocity dispersions of unresolved low mass stellar systems (e.g. the core of globular clusters), line asymmetry and detailed chemical composition of stars, can only be carried out with high resolution spectroscopic observations. Unfortunately, these studies are restricted to relatively bright objects due to the high spread of the light over the detector. However, the information about the Doppler shift, the velocity broadening and the metallicity of spectra, is in fact distributed among all the spectral lines and can be concentrated into a few parameters by a cross-correlation algorithm. If the number of lines is large enough, it is possible to extract information from low signal-to-noise $(\mathrm{S} / \mathrm{N})$ ratio spectra and to observe faint stars.

Griffin (1967) demonstrated that the optical cross-correlation technique with a mask, first described by Fellget (1953), was able to measure quickly and easily, accurate radial velocities with higher sensitivity than the conventional photographic technique. Ten years later, Baranne et al. (1979) extended this technique to echelle spectrographs by building the CORAVEL and showed that the metallicity could also be extracted from the cross-correlation functions (CCF).

Simkin (1974), Tonry and Davis (1979) and Sargent et al. (1977) gave the first detailed descriptions of correlation techniques to measure the Doppler shift and velocity broadening of digitized stellar spectra. With the advent of CCDs, these techniques have been intensively applied in several modifications and improvements (e.g. Bender 1990 for a review) to various resolutions and wavelength ranges. Recently, Dubath et al. (1990) applied the CORAVEL technique with success to high resolution CCD echelle spectra to measure small velocity broadening of composite spectra. 
The purpose of this paper is to describe in detail the CORAVEL technique applied to digitized spectra. At present, this technique is routinely available with the automatic reduction program of the ELODIE echelle spectrograph at the Observatoire de Haute-Provence in France

\section{THE CORAVEL-TYPE NUMERICAL CROSS-CORRELATION}

The main difference between the classical numerical cross-correlation techniques and the CORAVEL-type one, is the structure of the template: The CORAVEL-type one is composed of box-shaped emission lines, matching the typical lines of cool stars, selected to give the best CCF (e.g. Baranne et al. (1979)). However, this template is only efficient if the number of lines is statistically significant (about 1000 lines). Therefore, this technique is almost restricted to echelle spectra covering a large wavelength domain band and to spectra having a significant number of lines (e.g. spectra of cool stars).

Formally, the cross-correlation process can be written:

$$
\begin{aligned}
& C(\epsilon)=\frac{R(\epsilon)}{R(\infty)} \\
& R(\epsilon)=\int_{-\infty}^{+\infty} S(v) M(v-\epsilon) d v
\end{aligned}
$$

where $C$ is the CCF, $S$ the spectra and $M$ the template, both expressed in velocity space $v$. Thanks to the box-shape of the lines in $M$, Eq. (2) can be simplified to:

$$
R(\epsilon)=\sum_{i} \int_{-\epsilon+u_{i}-\Delta u_{i} / 2}^{-\epsilon+u_{i}+\Delta u_{i} / 2} S(v) d v
$$

where $\Delta u_{i}$ is the width of the $i^{\text {th }}$ box of the template centred on $u_{\mathrm{i}}$.

The spectrum is modeled by a set of absorption lines with Gaussian shapes. If only the $n_{l}$ lines matching the template at $\epsilon^{\prime}=\epsilon$ are considered, Eq. (3) can be written:

$$
R\left(\epsilon^{\prime}\right)=\sum_{i}^{n_{l}} \int_{-\epsilon^{\prime}-\Delta u_{i} / 2}^{-\epsilon^{\prime}+\Delta u_{i} / 2} F_{i}^{c}\left(1-D_{i} \exp \left(-.5 \frac{(v 2.355)^{2}}{w^{2}}\right)\right) d v
$$

where $w$ is the FWHM of the lines, $D$ their relative intensities and $F^{c}$ the Flux in the continuum of the spectra. If all the quantities are expressed in function of $w:(\alpha=\Delta u / w, \epsilon=$ $\epsilon^{\prime} / w$ and considering $\Delta u_{i} \equiv \Delta u$, Eq. (4) can be written:

$$
\begin{aligned}
R(\varepsilon) & =\sum_{i}^{n_{l}} R_{i}(\varepsilon) \\
R_{i}(\varepsilon) & =\int_{\varepsilon-\alpha_{i} / 2}^{\varepsilon+\alpha_{i} / 2} F_{i}^{c}\left(1-D_{i} \exp \left(-.5 x^{2}(2.355)^{2}\right)\right) d x .
\end{aligned}
$$

Finally, the assumption $\alpha_{\mathrm{i}} \equiv \alpha$ allows us to express $C(\epsilon)$ :

$$
C(\varepsilon)=1-\frac{G_{\alpha}(\varepsilon)}{\alpha}\langle D\rangle
$$


where

$$
\begin{aligned}
G_{\alpha}(\varepsilon) & =\int_{\varepsilon-\alpha / 2}^{\varepsilon+\alpha / 2} \exp \left(-2.77 x^{2}\right) d x \\
\langle D\rangle & =\frac{\sum_{i=1}^{n_{l}} F_{i}^{c} D_{i}}{\sum_{i=1}^{n_{l}} F_{i}^{c}} .
\end{aligned}
$$

The shape of the CCF is determined from the $G_{\alpha}$ function which depends on the intrinsic shape of the spectral lines and on the width of the template lines. The depth of the CCF is related to the average $\langle D\rangle$ of the depths of the spectral lines matching the template, weighted by the flux in the continuum of the spectrum. Therefore, it is possible to carry out a classic spectroscopic analysis on this "concentrated single line" and extract information concerning the spectrum itself (see Fig. 1a). Furthermore, thanks to the large number of blended lines and some template mismatchings, the shape of the CCFs are extremely well approximated by Gaussian functions. Howewer, if the spectral lines are strongly asymmetric, the CCF can significantly deviate from the Gaussian approximation (see Fig. 1b).
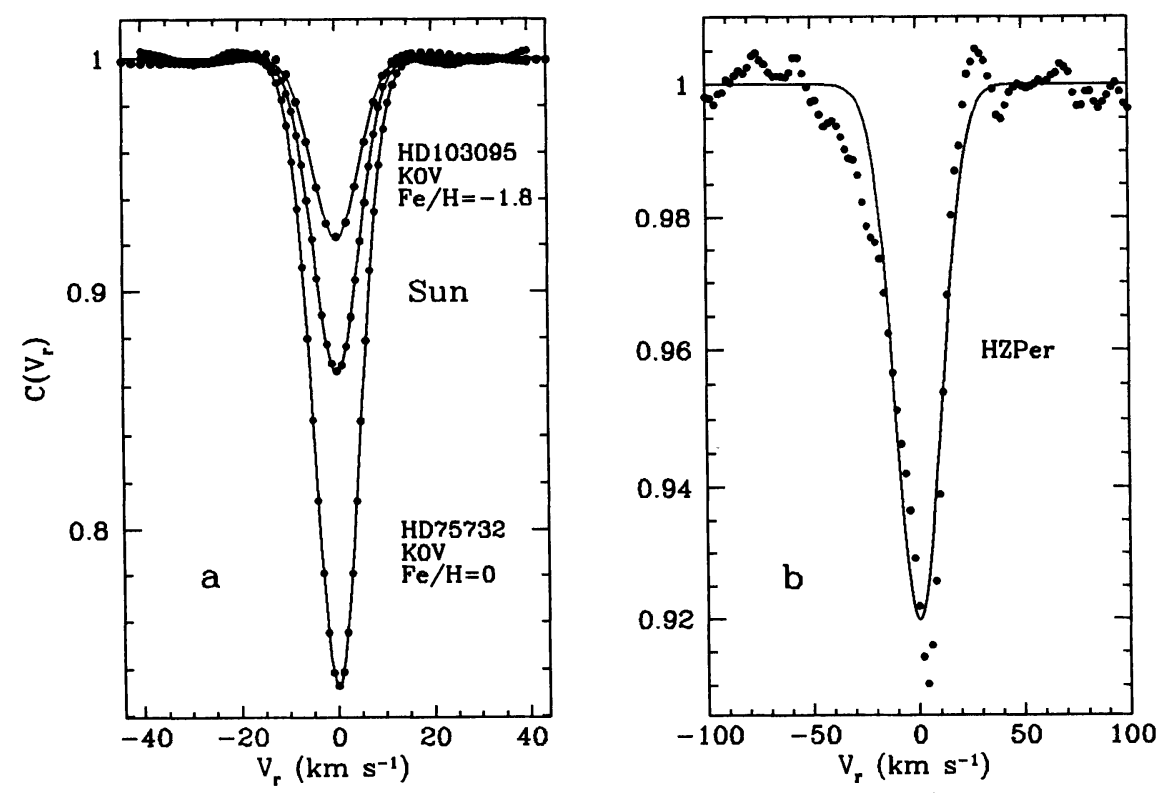

Fig. 1. CCFs for stars with various temperatures and metallicities (dots). The CCFs reproduce well the average behavior of the majority of lines matching the template (neutral lines). The solid lines represent the fitted Gaussians and it is worth noticing that they approximate the CCFs very well. b) CCF of a Cepheid star at a particular pulsating phase where the lines are asymmetric. 


\subsection{Computing the Error}

The estimation of the error on the fitted parameters of the Gaussian, due to photon and CCD read-out noise $\left(\epsilon_{p}\right)$, is done with the help of a numerical model. Numerous Monte-Carlo simulations have been carried out using Eq. (7) and the approximation of Gaussian-shaped spectral lines.

The following expression is derived for the rms of the radial velocity $\left(\epsilon_{p}\left(V_{\eta}\right)\right.$ with an uncertainty of $20 \%$ :

$$
\varepsilon_{p}\left(V_{r}\right)=.44 \frac{w_{i}}{D(S / N)\left(n_{l}\right)^{1 / 2}} O\left(s, s_{i}\right)\left(\mathrm{km} \mathrm{s}^{-1}\right)
$$

where $w_{\mathrm{i}}$ is the resolution (FWHM) of the spectrograph in $\mathrm{km} / \mathrm{s}, D$ the relative depth of the corresponding CCF without noise, $(S / N)$ the average signal-to-noise ratio of the spectrum, $n_{l}$ the number of spectral lines matching the template, $s$ the number of pixel per resolution element (FWHM) of the spectrum and $s_{i}$ the instrumental sampling per resolution element (in pixels). The function $O\left(s, s_{i}\right)$ is a correction to take account of oversampled spectra due to the velocity broadening $\left(s>s_{i}\right)$ and an intrinsic instrumental sampling greater than two: $O\left(s, s_{i}\right)=$ $1.6 s_{i}^{-1}+0.2\left(s / s_{i}\right)(2<s<10)$. It is worth mentioning that for unresolved spectra, observed with an instrumental sampling of two pixels per resolution element, $O\left(s, s_{i}\right)=1$.

The estimation of the rms on the fitted rms $(\sigma)$ of the Gaussian is described by the same equation as the radial velocity:

$$
\varepsilon_{p}(\sigma)=\varepsilon_{p}\left(V_{r}\right)
$$

Formally, the value of the error is independent of the $\alpha$ value of the template. The use of larger boxes for the template would reduce the depth of the CCF and the amplitude of the noise in a similar way, only leading to a scaling of the CCF. But the use of a template with $\alpha=1$ is optimum because it minimizes the blend effect and avoids an undersampling of the CCF. One can notice that the method of the Q-optimization of Baranne et al. (1979) always produces templates with $\alpha \approx 1$.

Equation (10) is only valid for a template with $\alpha=1$. However, as the depth of the CCF is a slow function of $\alpha$ (see Eqs.(7) and (8)), the equation can also be used with templates ranging from $\alpha=0.75$ to $\alpha=1.25$.

It is worth noticing that Eq. (10) reproduces the empirical Eq. (3) of Dubath et al. (1990) and is compatible with the result of Murdoch and Hearnshaw (1991) computed analytically for stellar templates.

\subsection{The Signal-to-Noise Limit}

The purpose of this paragraph is to estimate the minimum $(\mathrm{S} / \mathrm{N})$ of the spectra to clearly distinguish a true CCF from a spurious one due to the noise. A CCF is considered as true when $D>3.5 \sigma_{\mathrm{c}}$, where $\sigma_{\mathrm{c}}$ is the rms in the continuum of the CCF, formally at $\mathrm{C}(\infty)$.

The relation between the rms in the continuum of the $R$ function $\left(\sigma_{\mathrm{R}}\right)$ at $R(\infty)$ and the 
rms of the spectrum due to noise $\left(\sigma_{s}\right)$ is $\sigma_{\mathrm{R}}=\left(n_{l}\right)^{1 / 2}(\alpha s)^{1 / 2} \sigma_{s}$. The link between $\sigma_{\mathrm{R}}$ and the rms of $C(\infty)$ is given by $\sigma_{\mathrm{c}}=\sigma_{\mathrm{R}}\left(\alpha, s, n_{l} F^{\mathrm{c}}\right)^{-1}$, where $F^{c}$ is the flux per pixel in the continuum of the spectra. Finally, with the definition $(S / N)=F^{c} / \sigma^{s}$, the following expression describes the rms in the continuum of the CCF:

$$
\sigma_{c}=\frac{1}{(S / N)\left(n_{l} \alpha s\right)^{1 / 2}}
$$

Using the detection criterion $D>3.5 \sigma_{c}$, a minimum $(\mathrm{S} / \mathrm{N})$ can be defined (see Fig.(2) for illustration):

$$
(S / N)_{\min }=\frac{3.5}{D\left(n_{l} \alpha s\right)^{1 / 2}}
$$

The combination of Eqs.(13) and (10) leads to the definition of the maximal feasible error:

$$
\varepsilon_{p}^{\max }\left(V_{r}\right) \approx .13 w_{i}(s)^{1 / 2} O\left(s, s_{i}\right)\left(\mathrm{km} \mathrm{s}^{-1}\right) .
$$

Equation (14) indicates that, for every resolution, there is a typical maximal accuracy beyond which the CCF could be mistaken for a spurious CCF generated from the noise. For the optimization of the telescope time, the concept of the maximal error has strong consequences on the choice of the instruments. For example, an observing program requiring only an velocity accuracy of five $\mathrm{km} / \mathrm{s}$ should not be carried out with a spectrograph having a resolution greater than 10,000 because a larger integration time is needed to measure reliable velocities with unecessary higher accuracy (see Table 1).

\section{TABLE 1}

Maximal error for various resolutions

\begin{tabular}{llll}
\hline $\begin{array}{c}\text { Resolution } \\
\mathrm{R}\end{array}$ & $\begin{array}{c}w_{i} \\
(\mathrm{~km} / \mathrm{s})\end{array}$ & $\begin{array}{l}\epsilon_{\mathrm{p} \max }\left(V_{r}\right) \\
(\mathrm{km} / \mathrm{s})\end{array}$ & $\begin{array}{c}\delta(w) \\
(\mathrm{kms})\end{array}$ \\
\hline 10,000 & 30 & 5.5 & 30.7 \\
20,000 & 15 & 2.8 & 15.5 \\
40,000 & 07.5 & 1.4 & 07.8 \\
\hline
\end{tabular}

Table 1 shows the maximal error for various resolutions beyond which the CCF could be mistaken for a spurious one ( $s=2$ and $s_{i}=2$, are used). $\delta(w)$ is the typical maximal error on the velocity broadening measurement, taking a Gaussian broadening function: $\delta(w)=\left(w_{i}{ }^{2}-\left(w_{i}\right.\right.$ $\left.\left.+2.355 \epsilon_{p}(\sigma)^{\max }\right)^{2}\right)^{1 / 2}$.

\subsection{Observational Strategy}

To investigate the optimum instrumentation to measure radial velocities and velocity broadenings, we compute the ratio of the exposure times, for two instrumental resolutions ( $w_{0}$ and $w_{1}$ ) needed to reach the same accuracy. 


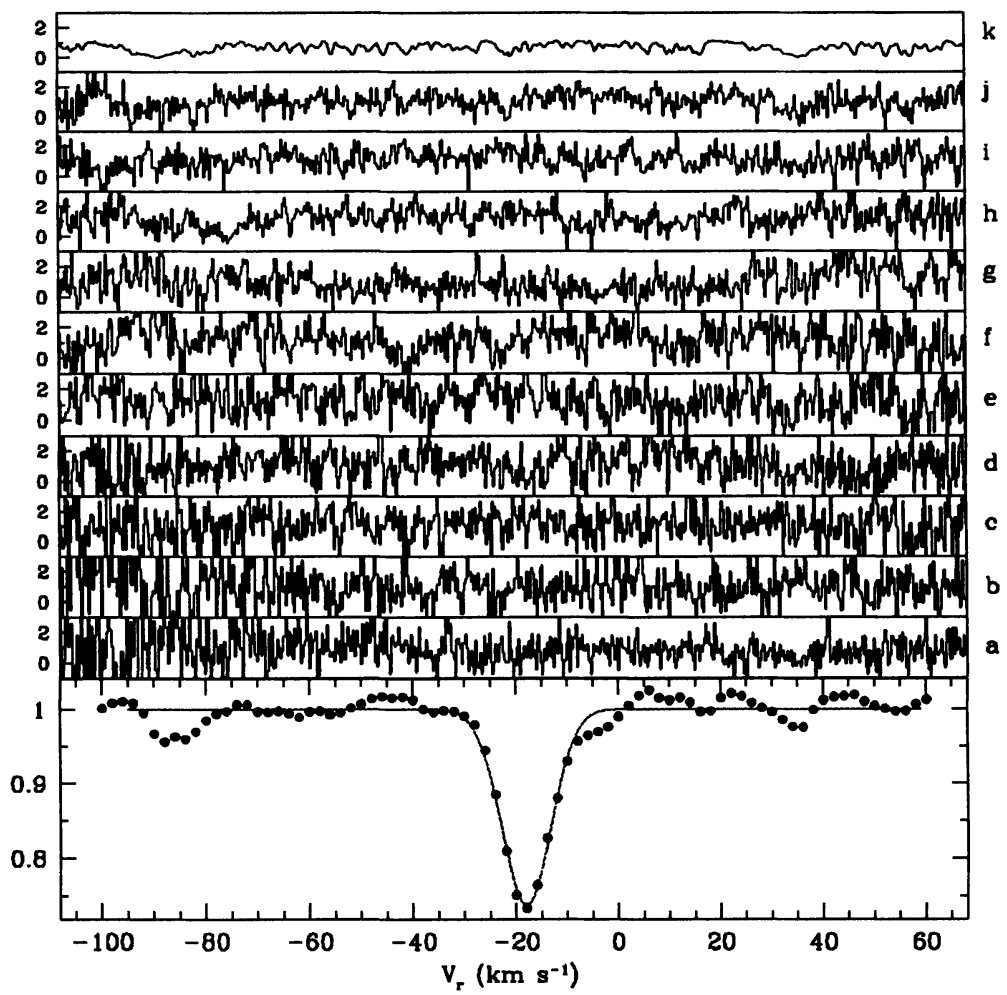

Fig. 2. Illustration of a CFF computed from a KO III spectrum with $(\mathrm{S} / \mathrm{N}) \approx 1$. The wavelength range used is from 4110 to $4440 \AA$ with a resolution of 40,000 . The number of spectrum lines matching the template is about 1000. The ten orders of this spectrum, covering $40 \AA$ each, are displayed from a to $j$. The last slice $(k)$ displays, for comparison, the same order as $j$ but at a higher signal-to-noise $(\mathrm{S} / \mathrm{N}=40)$. In the continuum of the CCF, we observe a typical noise of about 0.02 which corresponds to the expected value from Eq. (12). It is worth mentioning that, for this spectrum, the minimum $(\mathrm{S} / \mathrm{N})$ to detect a reliable CCF would be 0.3 (computed from Eq. (13)) 
For both resolutions we suppose that $(S / N)>(S / N)_{\min }$ and thus that the errors are lower that the maximal ones to measure reliable CCFs with the highest resolution.

Two noise regimes are considered: "read-out noise limited" (RONL) and "photon noise limited" (PNL). The first, (RONL), has a $\left.S / N \sim w_{i} i f\left(w_{i} / w_{s}\right) t\right)$ and the second, (PNL), has a $(S / N) \sim\left(w_{i} f\left(w_{i} / w_{s}\right) t\right)^{1 / 2}$. The function $f\left(w_{i} / w_{s}\right)$ is a transmission function to take into account the seeing effect, where $\mathrm{w}_{s}$ is the width of the seeing spot projected on the detector in velocity unit and $w_{i}$ the instrumental resolution. We use the factor given by Allen (1973) to convert the slit width $\left(w_{s l}\right)$ to the FWHM of the resolution: $\left(w_{s l} \approx 1.5 w_{i}\right)$. We suppose $w_{0}<w_{1}$. In the case both spectra are not resolved $\left(s=s_{i}\right)$, we have $O\left(s, s_{i}\right)=1$ and $D_{0}=\left(w_{1} / w_{0}\right) D_{1}$, then $w_{1}^{2} S / N_{0}$ $=w_{0}{ }^{2} S / N_{1}$. Therefore we find:

$$
\begin{gathered}
\frac{t_{0}}{t_{1}}=\frac{f\left(w_{1} / w_{s}\right)}{f\left(w_{0} / w_{s}\right)} \frac{w_{0}}{w_{1}} \quad \text { (in the RONL regime), } \\
\frac{t_{0}}{t_{1}}=\frac{f\left(w_{1} / w_{s}\right)}{f\left(w_{0} / w_{s}\right)}\left(\frac{w_{0}}{w_{1}}\right)^{3} \quad \text { (in the PNL regime), }
\end{gathered}
$$

If the spectrum is completely resolved by the instrumental resolution $w_{0}\left(s_{o}>s_{i}\right)$ and at the resolution limit with $w_{1}\left(s_{1}=s_{i}\right)$, we have $D_{0}=D_{1}$ and $O_{1}\left(s, s_{i}\right)=1$ and thus $w_{1}(S / N)_{0}=$ $w_{0}(S / N){ }_{1} O_{0}\left(s, s_{i}\right)$. Therefore we find:

$$
\begin{gathered}
\frac{t_{0}}{t_{1}}=\frac{f\left(w_{1} / w_{s}\right)}{f\left(w_{0} / w_{s}\right)} O_{0}\left(s, s_{i}\right) \quad \text { (in the RONL regime) } \\
\frac{t_{0}}{t_{1}}=\frac{f\left(w_{1} / w_{s}\right)}{f\left(w_{0} / w_{s}\right)} \frac{w_{0}}{w_{1}} O_{0}\left(s, s_{i}\right)^{2} \quad \text { (in the PNL regime) }
\end{gathered}
$$

In Fig. (3), the behavior of $t_{0} / t_{1}$ in these four cases are displayed for two typical seeing values. A general conclusion seems to come into view from these figures: The optimum is rather found towards the high resolutions than the lower ones, but the resolution of the spectrograph must never reach the intrinsic resolution of the spectrum. This behavior of the optimum seems to be independent of the seeing value if the slit width is reasonable compared to the seeing spot.

\section{REFERENCES}

Allen, C. W. 1973 Astrophysical Quantities, Athlone Press, London, Ch 4

Baranne, A., Mayor, M. and Poncet, J. L. 1979 Vistas in Astronomy, 23, 279

Bender, R. 1990 A\&A 229, 441

Dubath, P., Meylan, G., Mayor, M. and Magain, P. 1990 A\&A 239, 142

Fellget, P. 1953. Optica Acta 2, 9

Griffin, R. F. 1967 ApJ 148, 465

Murdoch, K. and Hearnshaw, J. B. 1991 A\&AS 186, 137

Sargent, W. L. W., Schechter, P. L., Boksenberg, A. and Shortridge, K. 1977 ApJ 212, 326

Simkin, S. M. 1974 A\&A 31, 129

Tonry, J. and Davis, M. 1979 AJ 84, 1511 

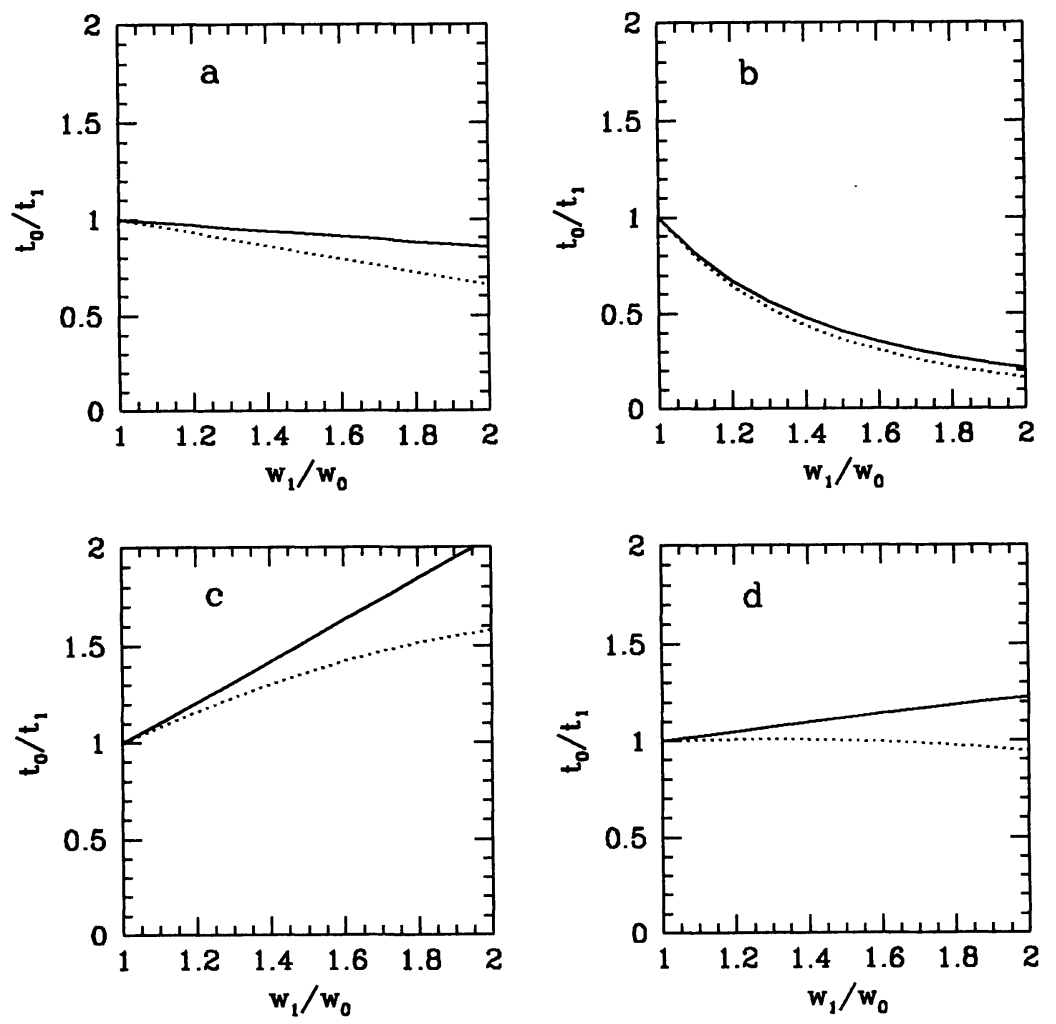

Fig. 3. The results from the Eqs.(15) in a, (16) in b, (17) in c, (18) in d are computed for two different seeing values: two and one times the slit width adjusted to the resolution $\mathrm{w}_{0}$ (solid and dotted line respectively). 


\section{DISCUSSION}

UNDERHILL: I suspect that it would be difficult to use this method with early type stars be cause the lines do not all have the same shape. Is it possible to work in the yellow-green and red regions where the re are numerous telluric lines and lines from diffuse interstellar bands superimposed on the stellar spectrum?

QUELOZ: You can work in any region you like. The only crucial point is having enough lines.

BIKMAEV: Is it possible to obtain simultaneously data on $\mathrm{Vr}$, Vsini and (or) asymmetry and metallicity from one frame?

QUELOZ: Yes, but you may need more than one template.

D'ODORICO: How sensitive is this technique to a changing line profile across the orders or across CCDs (changing focus, etc.)?

QUELOZ: It is sensitive. In fact, if the effect is clearly visible on high signal-to-noise spectra you will also see it by doing cross-correlation on restricted wavelength regions of poor signal-tonoise spectra.

CUBY: Why don't you use a more "realistic" template (synthetic spectrum) instead of boxed shape lines. The solution of the matched filter problem is to correlate with a function of similar shape as the one we want to extract.

QUELOZ: With the large number of lines used and the numerous blends present in cool stars, I do not think there is a big difference. But anyway, following P. Connes (1985, A\&AS), it seems that neither the boxes nor the Gaussians are optimized line shapes to extract the radial velocity. 